\title{
Formación y experiencia \\ en la universidad
}

\section{Artículo de reflexión}

Revista Colombiana de Educación, N. ${ }^{\circ} 70$. Primer semestre de 2016 Bogotá, Colombia.

\section{//Training and Experience \\ at the University}

\section{//Formação e experiência \\ na universidade}

\section{Alberto Martínez Boom*

\begin{abstract}
Profesor titular de la Universidad Pedagógica Nacional. Doctor en Filosofía y Ciencias de la Educación. Website: www.albertomartinezboom.com. Intervención preparada para el "Encuentro de Rectores y Directivos Académicos de universidades pedagógicas e instituciones formadoras de maestros en América Latina". Bogotá, 6 de octubre de 2015. El artículo es uno de los resultados del proyecto de investigación: "Balance del plan distrital de formación docente 2009-2012" financiado por el IDEP y la Universidad Pedagógica Nacional.
\end{abstract}

\section{Resumen}

Asistir a la crisis de la formación en la universidad actual denota para el autor una posibilidad de interrogación y de repensamiento sobre las posibilidades éticas y políticas del propio proyecto universitario. El artículo describe algunas de las mutaciones y cambios que sobre la formación y la experiencia han producido los derroteros de la sociedad del aprendizaje, pero no se complace con aceptarlos sino que intenta reflexionar sobre ellos desde un lugar singular: la cuestión del valor y la exigencia de dotarlas de valoración. Este punto de vista crítico sugiere un espacio para la conservación de tradiciones y prácticas susceptibles de legitimar el lugar social de la propia universidad.

\section{Abstract}

Attending the current education crisis at universities indicates a possibility for the author to question and rethink the ethical and political possibilities of their own university project. The article describes some of the mutations and changes in and experience that the paths of the learning society have produced, but it doesn't consent to accepting them it also tries to reflect on them from a single perspective: the question of value, and the need to provide an assessment. This critical point of view opens the space for the conservation of traditions and practices that could legitimize the social role of university itself.

\section{Resumo}

Assistir à crise da formação na universidade atual, denota para o autor uma possibilidade de interrogação e de repensamento sobre as possibilidades éticas e políticas do próprio projeto universitário. O artigo descreve algumas das mutações e mudanças que sobre a formação e a experiência hão produzido os caminhos da sociedade da aprendizagem, mas não se satisfaz com aceitá-los, mas que intenta refletir sobre eles desde um lugar singular: a questão do valor e a exigência de proporcionar-lhes valor. Este ponto de perspectiva crítica sugere um espaço para a conservação de tradições e práticas susceptíveis de legitimar o lugar social da própria universidade.

\section{Palabras clave}

Formación, experiencia, universidad, pedagogía, aprendizaje

\section{Keywords}

Formation, experience, university, pedagogy, learning

\section{Palavras chave}

Formação, experiência, universidade, pedagogia, aprendizagem 


\section{¿Qué pasa con la formación hoy en la universidad?}

En las últimas décadas la universidad ha perdido el monopolio que profesaba tanto sobre la formación como sobre la apropiación de las disciplinas. La formación parece haber dejado de ser el asunto central de la universidad y a cambio se ocupa de otras necesidades profesionales que exigen la participación de un aprendiz consumidor, el cual decide, en últimas, lo que cree necesitar o lo que le marca el deseo construido por la publicidad o por las ofertas tecnológicas. La formación, otrora objeto de la pedagogía, hoy es una nueva cosa en manos de la tecnología.

¿Por qué ocurre esto? En principio intento responder desde tres aristas: la masificación, la modernización educativa y la virtualización e ingreso del aprendizaje por competencias.

a. Las ideas y prácticas en torno a la formación encontraron obstáculos difíciles al tener que afrontar la educación de masas. Sistemáticamente interrogaron el quehacer de la universidad, juzgaron sus tradiciones como improductivas, memorísticas, derrochadoras de tiempo, holísticas, más interesadas en los conocimientos que en resolver los problemas de mercado actual y futuro. $Y$ sin mayor objeción a las nuevas demandas, la universidad aceptó sus exigencias hasta comprometerse con la "bien vista" cualificación de profesionales acorde a la economía del conocimiento, a los derroteros de la globalización y a las lógicas que hacen de la educación un objeto de crédito supremamente rentable para el mercado financiero. La universidad entra así en la lógica de la escolarización.

Desde la mundialización de la economía y de las comunicaciones se pusieron en marcha rápidamente transformaciones en los sistemas de escolarización. Dicha mundialización se materializó a través de prácticas que incluían cooperación técnica internacional, diagnósticos comunes, sistemas de información, consensos, declaraciones mundiales, etc. Estas prácticas impiden pensar la educación sin pasar por su mundialización, es decir, por la conformación (en la mayoría de países del mundo) de sistemas educativos isomórficos, cuyas similitudes y semejanzas pasan por la decisión de organizaciones internacionales encargadas de difundir una cultura educativa 
de efectos globales. De ahora en adelante mundializar la educación significaría convertirla en objetivo estratégico central de los mercados. Sin dejar de ser mercancía, la educación ingresa a un nuevo modo de ser que he denominado "edu-capital", entendido como una manera de ser de la educación en la que su función principal se define en función de la capitalización, por lo tanto se ubica en el orden empresarial y está ligado a los propósitos de aumento de la eficacia y el incremento de la ganancia. De este modo se supone que cada individuo puede ser capitalizable y que se puede incrementar su rendimiento de tal forma que el cerebro humano deviene capital ${ }^{1}$. Atrás parece haber quedado la idea de un Estado que asumía para sí el privilegio y la financiación de la educación. Aparece la exigencia de intervenir en la educación con la mayor

\footnotetext{
La noción de educapital, que he venido trabajando muestra que es a través de la modulación individual y de maximizar las capacidades de cada individuo para tomar elecciones en su propio interés como se produce el máximo beneficio para la sociedad, o mejor, para la economía. Esta actualización de la ética utilitaria muestra que existe una transacción constante entre lo individual y lo colectivo. Se trata de una nueva manera de gobernar, no a través de la sociedad sino a través del individuo y del uso de sus elecciones. El educapital pone en relación tres factores estratégicos: inclusión, capital y aprendizaje.
}

economía posible pasando del monopolio educativo del Estado a una oferta que se abre y se diversifica, incluso a escala trasnacional, conservando eso sí un papel garante, vigilante, evaluativo, regulador y desregulador. Este es el paradigma que advierte el paso del Estado docente al Estado evaluador.

Lo anterior no significa que la educación como razón de Estado desaparezca o que el Estado abandone prácticas específicas de intervención educativa sobre las poblaciones. Todo lo contrario, los Estados conservan, financian, racionalizan y focalizan su papel garante e incluyente en lo que se refiere a la educación de los sectores vulnerables, marginados y excluidos con el objetivo de que ingresen a la lógica de la escolarización y del aprender a aprender. Para ello construyen todo un discurso de política educativa que habla de equidad y de justicia. Pero esto tiene sus costos.

b. Además de la masificación, los procesos de modernización del nivel terciario serán puestos en crisis. El libro de Philip Coombs, Crisis mundial de la educación, es emblemático al respecto porque señala dos objetivos del discurso de la crisis: 
[...] el primero consiste en reunir los hechos básicos de una visible crisis mundial en el terreno de la educación, explicitar las tendencias inherentes a estos hechos, y sugerir los elementos de una táctica a seguir ante ellos. El segundo objetivo pretende presentar un método para enfocar el sistema de educación, no como una serie de piezas sueltas donde cada faceta es un fragmento aislado, sino como un sistema en el que cada parte actuante emite sus propias "señales" para advertir si la acción conjunta se relaciona de forma satisfactoria o no. (Coombs, 1971, p. 5)

Como se trata de sistemas en proceso de mutación se necesitan reformas para garantizar tanto la funcionalidad como la introducción de prescripciones universales capaces de optimizar el uso de nuevos medios formales y tecnológicos. Entre otras cosas porque la masificación requiere tecnologías diversificadas, exige usos capaces de responder a las contingencias de las tecnologías sociales y materiales. Todo un arsenal de planes y cambios que en palabras de Benjamin Levin (1998) conforman una epidemia política de reformas en la universidad globalizada. En suma, una vocación pragmática con efectos inconmensurables sobre lo que significa formar y ser profesor. Se indica que el tiempo de la enseñanza es valorado hoy como lento y derrochador; y en su defecto se recurre ahora a la velocidad del aprendizaje. Edgar Faure (1973) en Aprender a ser, la educación del futuro indica que "el acto de enseñar cede paso al acto de aprender", y supera la concepción de una educación limitada en el tiempo y encerrada en el espacio. Semejante transformación en el énfasis de las actividades educativas hace emerger modelos mucho más flexibles y diversificados, modelos que tendrán como característica la ampliación ad infinitum de la capacitación permanente y continua. Y esto lo aceptamos sin la menor cítica. El tesoro del que habla el Informe Delors (1996) ya no consiste en la formación de un ciudadano sino de un sujeto, que a partir de ahora podrá hacerse cargo de sus propias necesidades y expectativas de aprendizaje en un mundo altamente competitivo. Aprender a competir pone en escena nuestra existencia en un mundo mucho más complejo y, por cierto, cada vez más hostil. Esta variación 
introduce aumento de la eficacia, la eficiencia y la pertinencia. Estos tres aspectos se agrupan en un término genérico: calidad de la educación. Este descentramiento exige del profesor un acto de abandono: privarse de su otrora experiencia respecto de la enseñanza y por supuesto de la formación. Ya no sorprende que nuestra inteligencia haya convertido la aspiración a la formación en prácticas de aprendizaje, en gestión y competencias de profesionalización, en administración de un simulacro cuyo valor no se opone a las tradiciones formativas. La idea de la universidad como un refugio en donde el saber madura lejos de las preocupaciones de la vida cotidiana y al cual se acude para beber en el recinto de la sabiduría parece haberse vuelto anacrónica.

c. Expresiones como "aprendizaje apoyado en la web", "tele aprendizaje", e-learning, "aprendizaje electrónico", "edu-entretenimiento", entre otros, describen una variación sustantiva del tiempo y del espacio en el que contemporáneamente se realiza la acción educativa.
La antigua formación parece haber devenido en nuevos procedimientos, nombrados por los expertos como "modelos eficientes", dada su capacidad de resolver aspectos puntuales de las viejas rutinas, es decir, tecnologías de la velocidad, técnicas de la transmisión, tecnologías de acción a distancia. Una tecnología es mucho más que equipos y técnicas, se trata de un acoplamiento de relaciones sociales y humanas en el que los equipos y las técnicas son tan solo un elemento. Según Nikolas Rose una tecnología alude a un conjunto estructurado por una racionalidad práctica gobernada por un objetivo más o menos consciente; es decir, ensambles híbridos de conocimientos, instrumentos, personas, sistemas de juicio, edificios y espacios, sustentados por niveles programáticos (Rose, 2012). Todo este modelamiento implica modelos educativos de innovación; eficacia y pertinencia; inclusión y equidad; autoaprendizaje; y monitoreo y evaluación permanente.

Modelos educativos de innovación. Por medio de la tecnología es posible integrar enfoques constructivistas con aprendizaje social, en razón de sus atributos de interactividad que apuntan a la producción de comunidades de aprendizaje. Las nuevas tecnologías ejercen un poder de motivación fundamental para el proceso de aprendizaje de los individuos. 
Eficiencia y pertinencia. Las nuevas tecnologías prometen construir conocimiento pertinente y actualizado, es decir que los viejos contenidos e incluso los otrora currículos se pueden actualizar en términos de virtualización: contenidos virtuales de aprendizaje.

Inclusión y equidad. El acceso mediático es en sí mismo inclusivo. Miles de personas pueden tener acceso a un mismo contenido educativo. La cuestión pasa entonces por el diseño, la producción, la distribución y el uso de estos contenidos.

Autoaprendizaje. Las nuevas tecnologías permiten la personalización de los aprendizajes. En cada individuo existe una facultad creativa virtual susceptible de ser acumulada como competencia individual y vinculada con el trabajo inmaterial, hecho que acentúa el carácter cognoscitivo de las tareas. En esta dirección se ha llevado a cabo una revitalización de didácticas y pedagogías tecnicistas y el consumo de tecnologías que permanentemente cambian, de modo que los alumnos consumidores desarrollan nuevas necesidades y mayores competencias. El individuo del autoaprendizaje ya no se deja alumnizar ni someter a lo que algunos han denominado "pedagogías del aburrido".

Monitoreo y evaluación permanente. Tanto por sus usuarios como por sus gestores y participantes, las nuevas tecnologías parecen diversificar sus recursos educativos al tiempo que permiten el monitoreo, control y seguimiento permanente, ya no de los resultados sino de sus procesos, es decir, de la gestión.

\section{Tensión en la formación: maestro o docente}

La distancia que habría entre el maestro y el docente diría que el primero se coloca en proximidad a la enseñanza, y el segundo lindaría con las lógicas y demandas del aprendizaje. Asi, la enseñanza que en otra época era definitoria para nombrar al maestro, hoy tiende a reemplazarse por una función llamada función docente, que puede cumplir cualquier individuo, en muchas instancias, y a través de varios dispositivos, sin duda ese tránsito entre el maestro y la función docente de hoy termina por oscurecerlo o, si lo prefieren, enrarecerlo. Incluso su diseminación es por exceso, no por defecto; tantos son docentes y hay docencia en todo, que ya nadie recuerda su especificidad. Se evidencia una ambigüedad que somete a los sujetos que enseñan a una cierta esquizofrenia.Sujetos ambiguos que transitan en una región de sombras donde se desubican y al mismo tiempo se multiplican en 
tantos otros que su presencia se hace superficial y hasta anodina. También hay quienes descartan definitivamente al enseñante y lo reemplazan por un sujeto profesionalizado o también los que lo recomponen como un experto en resolución de problemas y otras habilidades multiusos. Separado de la enseñanza, su papel se recompone al definirse que su tarea en el proceso de la educación es servir de facilitador de aprendizajes. El énfasis en el aprendizaje señala una desesperación secreta en el valor de lo eficaz, lo inmediato y lo contrastable. Ahora, con la docencia virtual, el modo de ser de los conocimientos supone otro tipo de mediaciones y mediatizaciones cuyos efectos modifican tanto la enseñanza de los conocimientos como la autoridad que antes parecía legitimarlo. Lejos de criticar la informática y la tecnología, habría que interrogar la manera como el profesor se hace funcional a ellas, cuando el proceso sería más potente al revés.

\section{Pensar la formación en planos y desde la experiencia}

Ninguna de las propuestas que hoy se plantean como innovadoras o radicalmente transformadoras se enfrentan a los desafíos de apostar por la experiencia del profesor. Hacerlo encarna diversas dificultades, por lo que se hace necesario sustentar este planteamiento. La gama de actores que intervienen en los llamados procesos de "formación docente" es amplia: organismos internacionales, gobiernos, academias, instituciones y sindicatos. Podemos metodológicamente pensar la "formación docente" como un dispositivo de relaciones, o mejor, un conjunto multilineal de fuerzas de diferente naturaleza, que son heterogéneas que siguen direcciones diferentes, que forman procesos en desequilibrio. Schérer Ilama a esto dispositivo, es decir, "Io que organiza, distribuye, distingue o reúne elementos, lo que vuelve inteligible un conjunto confuso" (2005, p. 252). Con esta disposición podemos pensar la formación del maestro como sometida a líneas de fuerzas, a posiciones, enunciados y relaciones que actúan como vectores, como tensores (Martínez Boom, 2012). En otras palabras, si lo que importa es una formación que afirme al maestro frente a los nuevos problemas que tocan a la puerta, nos corresponde una labor de construcción y análisis de dicha formación como dispositivo capaz de leer diversas tensiones al quebrar la lógica del plan en favor de un trabajo desde planos, o mejor de una unidad trascendente a una pluralidad inmanente. Los planos se distinguen del plan. Un plan es una herramienta de planificación, como tal, es trascendente y pretende anticipar el futuro, ordenarlo, prever su advenimiento; de ahí su formulación anual, quinquenal o decenal. Los planos, por el contrario, son inmanentes, cartográficos y concretos, y reconocen la subjetividad de diversos actores. Pensar el problema 
de la formación de maestros en función de planos es darle a cada plano ritmo, velocidad y tiempo diferentes. Lo que precisamente interesa del análisis por planos son sus posibilidades diferenciadoras, relacionales y problematizadoras. De esta manera el plano del sistema educativo reconoce la formación de maestros como lo que se requiere para el funcionamiento del propio sistema, circunstancia bien diferente cuando se piensan las prácticas de formación en las dinámicas de lo institucional, o desde las experiencias de un entusiasmo que es capaz de afectar las prácticas formativas pero que se ubica en un plano exterior, fronterizo, externo a los desafíos y necesidades del sistema. Lo anterior no quiere decir que el ejercicio formativo visto desde lo no convencional sugiera caos, falta de rigor o que lo sustituya cualquier cosa; todo lo contrario, importa la regulación formativa del orden sistémico, tanto como las posibilidades afirmativas de lo que este orden no controla. Pensar la formación docente desde esta geometría permite otro tipo de relaciones: el plano de las instituciones, el plano de los saberes, el plano de los sujetos y el plano de los bordes (Martínez Boom et al, 2015, 31-34).

\section{Formación desde la experiencia}

Pensadores como John Dewey habían imaginado para la cultura un puente pragmático entre educación, experiencia y democracia que, grosso modo, podemos apropiar así: "aprender por la experiencia es establecer una conexión hacia atrás y hacia adelante entre lo que nosotros hacemos a las cosas y lo que gozamos o sufrimos de las cosas, como consecuencia" (Dewey, 1995, p. 125). Es la falta de trabajo con la experiencia lo que explica tanto la pobreza de la formación como el malestar docente, la pérdida de autoridad del maestro, el exceso de productivismo que nos lleva a "la sociedad del cansancio", la inconformidad y el sinsentido en las labores cotidianas. Eliminar la experiencia es el propósito central de varias propuestas actuales. Y es que un maestro sin experiencia se compromete rápidamente con el aprender a aprender, con el facilismo de lo que hoy podemos nombrar como "pedagogías fashion" o "pedagogías light". Un maestro desprovisto de experiencia pierde valor, acepta sin crítica la educación centrada en competencias. La eliminación de la formación pedagógica como requisito para el ejercicio docente 
no valoriza al maestro sino que lo funcionaliza ${ }^{2}$. La experiencia no está dada; nombrarla exige quebrar toda posible ingenuidad sobre la formación o sobre la identidad del maestro, para componer los trazos de unas experiencias en plural, presentes en discursos teóricos, institucionales y prácticos, susceptibles de configurar diagnósticos, trayectos y apuestas afirmativas sobre lo múltiple y diverso de su devenir. Aun cuando el hecho de vivir, estudiar o pasar por una institución produzca efectos, esa estancia no necesariamente constituye una experiencia: la mera actividad no constituye experiencia. Para que esta se produzca se requiere aprehenderla como saber, es decir, que se incorpore como acto de pensamiento.Pensar la experiencia para repensar la formación pasaría por discutir mínimamente tres asuntos: el valor de la experiencia; la articulación de la experiencia con la formación; y la experiencia como posibilidad para el pensamiento y el acontecimiento. Voy a poner en valor la formación.

2 Al efecto trabajos de Walter Benjamin y Giorgio Agamben han anunciado que en nuestra época "ya nadie parece disponer de autoridad suficiente para garantizar una experiencia" (Agamben, 2007, p. 9), "cualquier discurso sobre la experiencia debe partir de la constatación de que ya no es algo realizable". Esta novedad requiere matización. Benjamin constata que las personas que iban a la guerra regresaban mudas del campo de batalla, es decir, no enriquecidas sino más pobres en cuanto a experiencia comunicable. Al interrogar por lo que había sucedido con la experiencia, Benjamin (1933) muestra cómo su pobreza y su crisis es un signo que define nuestra época.

\section{El valor de la experiencia}

Si suponemos que la experiencia pedagógica del maestro se construye, que no la configura el simple paso por la institución, que no son los años de permanencia en un cargo o la acción rutinaria los que nos dotan de experiencia, se hace necesario entonces direccionar los actos de formación hacia este valor de la configuración de la experiencia pedagógica del maestro, en cuanto sujeto individual y colectivo.

Lo que interesa de la experiencia es el valor que agrega, su destello único y singular. La experiencia se dota de valor porque contiene la ampliación del horizonte conceptual a través del reconocimiento de los propios problemas en la búsqueda de soluciones y alternativas ${ }^{3}$.Sin embargo, configurar la experiencia, hacerla sonar y reivindicarla dista mucho de los lenguajes reduccionistas de quienes dicen hacer sistematización de experiencias. Descontaminar la palabra experiencia de estas simulaciones significa dejar de aplanarla en los lenguajes del "conocimiento alternativo" y de su espontaneísmo subalterno. Se trata de no hacer de la experiencia "una cosa, de no objetivarla, no cosificarla, no homogeneizarla, no calcularla, no hacerla previsible, no fabricarla, no pretender pensarla científicamente o producirla técnicamente" (Larrosa, 2003, p. 4).

\footnotetext{
3 La experiencia no es productiva sino valorativa, "dotar de valor" no es igual a adquisición, no es algo que se transfiere; el valor pedagógico es intrínseco a la configuración de la experiencia de maestro porque le exige asumir posición frente a hechos y propuestas, le exige saber, construir respuestas y saberes propios.
} 


\section{Experiencia y formación}

Una experiencia genuinamente formativa tendría el efecto de incitar la curiosidad, fortalecer la iniciativa y generar deseos lo suficientemente intensos como para permitirse abordar con éxito en el futuro los obstáculos y problemas propios de cualquier experiencia, de ahí que la experiencia pedagógica haría más asequibles, más significativas y más controlables las experiencias posteriores. "El problema central de una educación basada en la experiencia es seleccionar aquel género de experiencias presentes que vivan fructífera y creadoramente en las experiencias subsiguientes" (Dewey, 2004, p. 73). Nombrar la clásica relación de la experiencia con la formación significa reiterar que la experiencia es

[...] lo que me pasa y lo que, al pasarme, me forma o me transforma, me constituye, me hace como soy, marca mi manera de ser, configura mi persona y mi personalidad. Por eso el sujeto de la formación no es el sujeto de la educación o del aprendizaje sino el sujeto de la experiencia: es la experiencia la que forma, la que nos hace como somos, la que transforma lo que somos y lo convierte en otra cosa. (Larrosa, 2003, p. 7)

En esto se encuentran pedagogía y formación, en la puesta en juego de una serie de conceptos para orientar la práctica pedagógica y valorar sus efectos, de forma que se vaya reformulando y reconstruyendo permanentemente. Es por la insistencia en la experiencia que podemos problematizar la autoridad pedagógica del maestro y su relación asimétrica con los estudiantes. Si la experiencia deviene en formación subjetiva es también una fuente de producción de saber.

Cuando la formación apuesta por construir la experiencia del maestro, cualquiera que sea el umbral en que aquella se desarroIle, se convierte en una disposición ética y cultural que le ayuda a movilizar su pensamiento, a pensarse de otras maneras y a asumir su quehacer como sujeto potente; esta posición lo distancia de su rol como simple funcionario. 


\section{Pensamiento y acontecimiento}

La formación orientada a construir la experiencia del maestro produce "afectación" ${ }^{4}$. La experiencia se conecta y se realiza en sintonía con el acto de pensar. El pensamiento es el articulador entre experiencia y pedagogía; es a partir de actos de pensamiento que el maestro pregunta, discute, se interroga, arriesga, problematiza y configura su experiencia. Como dice Dewey: "ninguna experiencia con sentido es posible sin algún elemento del pensamiento" (1995, p. 128); pensar traduciría un "esfuerzo intencional para descubrir las conexiones especificas entre algo que nosotros hacemos y las consecuencias que resultan, de modo que ambas cosas lleguen a ser continuas" (1995, p. 129).La experiencia articula razón y acción, espíritu y cuerpo. Está atravesada por los sentidos y el

4 Formaría parte de la política de formación de maestros el esfuerzo de regulación biopolítica de aquello que se busca transformar, porque parece propio de los dispositivos biopolíticos la regulación experta, tanto de la educación como de los discursos sobre el aprendizaje, la cognición, la innovación, las inteligencias, la creatividad, las competencias, la cooperación entre cerebros, y por supuesto, de la formación de maestros; es decir, de regímenes que regulan, diferencian, articulan, generalizan, individualizan conforme a un sentido amplio de economía política. El acontecimiento opera como impulso para salirse de lo cotidiano, transgrede la rutina y se arriesga a lo impensado. Para Larrosa, la experiencia es siempre nuestra experiencia, lo que nos acontece a nosotros, y lo que nos acontece en el tiempo de nuestras vidas [...]. No sólo porque la experiencia es un acontecimiento para nosotros, sino porque requiere de nosotros una apertura, una capacidad de ser afectados; y porque nos solicita, a veces, una respuesta" (1996, p. 468) pensamiento, pero no es solo experimentar como ensayo y error, se trata de experienciar: sentir, vivir, sufrir, reflexionar los hechos, repensarlos hasta convertirlos en experiencia vital $^{5}$. Estas precisiones permiten afirmar que las experiencias pedagógicas son singulares, distinción que establece distancias con las llamadas "innovaciones educativas" o "experiencias significativas" que buscan replicarse masivamente en cualquier lugar y con actores que no mantienen ninguna relación con la experiencia.

Para terminar, habría que señalar que

La educación exige de nosotros algo del orden de la inauguración de la novedad. Inaugurar novedad no implica renunciar a lo antiguo ni desconocer la historia. Simplemente habilitarnos a pensar que quizá haya otros modos posibles, Ilevamos mucho tiempo de historia pensando que hay un solo camino, una única respuesta. (Frigerio, 2004, p. 5)

$\mathrm{O}$ incluso creyendo que podemos limitar el pensamiento a anclarnos en los dualismos y las dicotomías que nos reducen a un modo de proceder

5 Para Dewey: "... lo opuesto a la acción reflexiva es, una vez más, la rutina y la conducta caprichosa. La primera acepta lo acostumbrado como una plena medida de posibilidad y deja de tener en cuenta las conexiones de las cosas particulares hechas. La última hace del acto momentáneo una medida de valor e ignora las conexiones de nuestra acción personal con las energías del ambiente [...] la reflexión en la aceptación de tal responsabilidad" (1995, p. 129) 
algorítmico. Pensar como un ejercicio vital, en movimiento para descolocarnos es algo que siempre es posible, sobre todo en instituciones como la universidad. Esa es la potencia de la universidad, que la diferencia radicalmente del nivel de la escolarización Ilamada educación terciaria.

Las universidades pedagógicas no resuelven el asunto de la formación con un didactismo pragmático y facilitador. Es necesario hablar de la formación, insistir en ella, traspasar su balbuceo para asumir el campo de formación como propuesta que articula lo que hacemos: planes de estudio, apropiación del mundo social y cultural, relación orgánica con distintos actores.

La existencia de la universidad se justifica al ser capaz de sostener, insistir y mantener un compromiso activo (afirmativo) con la formación, es decir, con su propia experiencia de formación. En esto radica su vitalidad, olvidarlo supone aniquilar la universidad (oscurecerla, enrarecerla, deslegitimarla). Los lenguajes actuales, rutinarios y atrapantes de los desafíos, de las competencias, de la acreditación, de la innovación expresan una misma desesperación que reduce, simplifica, homogeniza y desarraiga el trabajo con la formación. Así, el lenguaje de la innovación aminora la creatividad, tanto como los simulacros repetitivos de la acreditación aíslan y dividen la tarea de formación. No imagino una violencia mayor para la propia universidad. No hay exageración en esta forma de violencia, tan técnicamente diseñada; sus efectos son claros: destruye la universidad como comunidad, aísla su lenguaje, imposibilita toda cercanía, y, por supuesto, también imposibilita el cuidado de sí como aspecto ético y estético.

\section{Referencias bibliográficas}

Agamben, G. (2007). Infancia e historia. Buenos Aires: Adriana Hidalgo Editora.

Benjamin, W. (1933). Experiencia y pobreza. En: Discursos interumpidos I. Madrid: Taurus.

Coombs, P. (1971). Crisis mundial de la educación. Barcelona: Península.

Delor's, J. et al. (1996). La educación encierra un tesoro. Madrid: Santillana - Unesco.

Dewey, J. (1995). Educación y democracia. Madrid: Morata

Dewey, J. (2004). Experiencia y educación. Bogotá: Nueva.

Frigerio, G. (2004). "La complejidad de educar". En: Revista Novedades Educativas, 168. Buenos Aires: Novedades Educativas. 
Faure, E. et al. (1973). Aprender a ser. Madrid: Alianza - Unesco.

Larrosa, J. (1996). La experiencia de la lectura. Estudios sobre literatura y formación. México: Fondo de Cultura Económica.

Larrosa, J. (2003). "La experiencia y sus lenguajes". Conferencia dictada en la Serie Encuentros y Seminarios, Barcelona, Departamento de Teoría e Historia de la Educación, Universidad de Barcelona. 11 páginas.

Levin, B. (1998). An epidemic of education policy: what can we learn for each other? Comparative Education, 34(2), pp. 131-142.
Martínez Boom, A. (2012). Verdades y mentiras sobre la escuela. Bogotá: IDEP.

Martínez Boom, A.; Martínez Pineda, M.C.; Calvo, G.; Soler, C. y Prada, M. (2015). Pensar la formación de maestros hoy. Una propuesta desde la experiencia pedagógica. Bogotá: IDEP.

Rose, N. (2012). Biopolítica de la vida. La Plata: Universidad Nacional de la Plata.

Schérer, R. (2005). A su disposición. En: Foucault, La pedagogía y la educación. Bogotá: Magisterio. 